\title{
Estudio de la reactividad entre aleaciones de aluminio y partículas de $\mathrm{SiC}$
}

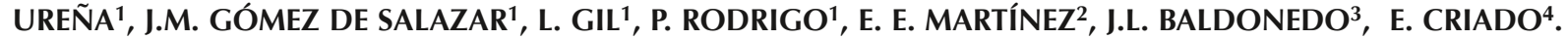 \\ ${ }^{1}$ Dpto. Ciencia de Materiales e Ingeniería Metalúrgica. Facultad de CC. Químicas. Universidad Complutense de Madrid. \\ ${ }^{2}$ Dpto. de Materiales Metálicos y Cerámicos. Instituto de Investigaciones en Materiales de la Universidad Autónoma de México. \\ ${ }^{3}$ Centro de Microscopía "Luis Bru". Universidad Complutense de Madrid. \\ ${ }^{4}$ Instituto de Cerámica y Vidrio. CSIC. Arganda del Rey. Madrid
}

\begin{abstract}
Las partículas de refuerzo de $\mathrm{SiC}$ reaccionan con el aluminio fundido y forman $\mathrm{Al}_{4} \mathrm{C}_{3}$ que es frágil y sensible al contacto con la humedad, lo que degrada las propiedades de los materiales compuestos constituidos por ambos componentes. La reacción en la intercara $\mathrm{Al} / \mathrm{SiC}$ depende de varios parámetros de fabricación como temperatura, tiempo de residencia, atmósfera y composición química, tanto de la matriz de aluminio como del refuerzo de $\mathrm{SiC}$.

Se han realizado estudios sistemáticos de reactividad entre partículas de $\mathrm{SiC}$ y aluminio fundido, analizando el efecto que tiene la formación de una capa de $\mathrm{SiO}_{2}$ por oxidación de las partículas cerámicas, antes de su incorporación en el fundido, como barrera protectora para prevenir el ataque del SiC por la matriz. Se ha estudiado también el efecto de la composición de la matriz metálica en dicha reactividad, empleando para ello varias aleaciones de aluminio: AA1170 (99,7\%Al), A-319.0 (Al-6Si-3Cu) y A-332.0 (Al-11Si-1,5Cu). Los materiales compuestos se prepararon mediante mezcla de partículas de SiC (30 $\%$ vol.) y polvo de las aleaciones de aluminio, prensado en frío y posterior fusión a $900^{\circ} \mathrm{C}$, variando el tiempo de residencia dentro del horno para simular un proceso de fabricación por colada. Los resultados obtenidos se comparan con los obtenidos para materiales compuestos del tipo AA 2014/SiC/20p.
\end{abstract}

Palabras clave: SiC, partículas, aluminio, materiales compuestos, reactividad, barrera de óxido.

Study on the reactivity between aluminium alloys and SiC particles.

The $\mathrm{SiC}$ reinforcement particles react with the molten aluminium to form $\mathrm{Al}_{4} \mathrm{C}_{3}$ that is brittle and sensitive to the the humidity. This reaction degrades the properties of the $\mathrm{SiC}$ reinforced aluminium matrix composites. The extend of the reaction in the $\mathrm{Al} / \mathrm{SiC}$ interface depends on the fabrication parameters such as the temperature, residence time, atmosphere and chemical composition of the aluminium matrix and the reinforcement.

Systematic studies on the reactivity between the $\mathrm{SiC}$ particles and molten aluminium alloys have been performed, analysing the effect of the presence of a $\mathrm{SiO}_{2}$ layer, which was formed by oxidation on the ceramic particles before their incorporation into the melt, as a protective barrier for preventing the attack of the $\mathrm{SiC}$. As well, the effect of the metal matrix composition on the interfacial reactivity has been studied employing different aluminium alloys: AA1070 (99,7\% Al), A-319.0 (Al-6Si-3Cu) and A-332.0 (Al-11Si-1,5Cu). The composites were prepared by mixture of SiC particles (30\% vol. \%), cold pressure and fusion at $900{ }^{\circ} \mathrm{C}$, varying the residence time in the furnace, which simulated a cast fabrication process. The results are compared with those obtained for a metal matrix composite (AA2014/SiC/13p).

Keywords: SiC, particles, aluminium, composites, reactivity, oxide barrier.

\section{INTRODUCCIÓN}

Los materiales compuestos de matriz de aluminio (AMCs) reforzados con partículas cerámicas son materiales de gran interés para muchas aplicaciones, tanto por sus elevadas propiedades mecánicas debidas a su mejorada relación resistencia-peso, respecto a las aleaciones no reforzadas, como por su capacidad para mantener su comportamiento en ambientes agresivos o a alta temperatura (1-2). La combinación del refuerzo cerámico, capaz de soportar cargas elevadas, con una matriz ligera les proporciona estas interesantes propiedades. Por otro lado, la posibilidad de aplicar técnicas convencionales de procesado, propias de aleaciones no reforzadas, supone un importante ahorro en costes de fabricación, si se compara con los de materiales reforzados con fibras continuas (3).
Sin embargo, los AMCs con refuerzo discontinuo, al igual que los reforzados con fibras cerámicas, pueden presentar problemas interfaciales por interacción química entre sus dos constituyentes principales $(4,5)$. Aunque este tipo de problemas pueden solventarse o atenuarse mediante la aplicación de barreras adecuadas sobre el refuerzo, las tecnologías de las que en la actualidad se dispone para su obtención sólo son compatibles y económicamente rentables en caso de ser aplicadas a refuerzos continuos (6).

En general, en un material compuesto de matriz metálica, la intercara entre la matriz y el refuerzo condiciona muchas de sus propiedades, entre ellas las mecánicas, ya que determina la forma de transferencia de carga entre ambos constituyen- 
tes. Como consecuencia de esto, en la mayoría de los casos, es deseable una intercara resistente a fin de conseguir un material compuesto con óptimas propiedades (7). Los procesos de fabricación disponibles para los AMCs implican dos posibles tipos de mecanismos de formación de las intercaras metal/cerámica presentes en el material: sólido-sólido y sólido-líquido. El presente trabajo se centra en el estudio de las intercaras formadas por mecanismos sólido-líquido, tal y como ocurre durante los procesos de fabricación por métodos de colada (compocasting, infiltración a vacío, forja líquida, etc.) que son los más ventajosos desde el punto de vista económico ${ }^{[1]}$.

Uno de los sistemas aluminio/ cerámico que posee mayores problemas de reactividad es el $\mathrm{Al} /$ $\mathrm{SiC}$, que es uno de los más utilizados en la fabricación de AMCs con refuerzo discontinuo. Estos materiales poseen una alta tendencia a la formación espontánea de $\mathrm{Al}_{4} \mathrm{C}_{3}$, durante los procesos de fabricación que impliquen la fusión de la matriz, por la reacción (8):

$$
3 \mathrm{SiC}(\mathrm{s})+4 \mathrm{Al}(\mathrm{l}) \rightarrow \mathrm{Al}_{4} \mathrm{C}_{3}(\mathrm{~s})+3 \mathrm{Si}(\mathrm{s}) \quad<1>
$$

La variación de energía libre para la reacción $<1>$ viene dada por la siguiente ecuación (9):

$$
\begin{aligned}
\mathrm{AG}\left(\mathrm{Jmol}^{-1}\right)= & 113.900-12.06 \mathrm{TLnT}+8.9210^{-3} \mathrm{~T}^{2+} \quad<2> \\
& +7.5310^{-4} \mathrm{~T}^{-1}+21.5 \mathrm{~T}+3 \operatorname{RTLn~}_{(\mathrm{Si})}
\end{aligned}
$$

donde $\mathrm{a}_{(\mathrm{Si})}$ es la actividad del $\mathrm{Si}$ en $\mathrm{Al}$ fundido, $\mathrm{R}$ la constante de los gases y T la temperatura absoluta. De acuerdo con esta ecuación, la energía libre de la reacción se hace negativa cuando la temperatura excede $\operatorname{los} 727^{\circ} \mathrm{C}(1000 \mathrm{~K})$; hecho que ocurre comúnmente en todos los procesos de fabricación en fase líquida disponibles para este tipo de materiales.

La formación de $\mathrm{Al}_{4} \mathrm{C}_{3}$ es altamente perjudicial por varias razones: 1) su presencia está asociada a un descenso de las propiedades mecánicas del material, no sólo porque se trate de un producto frágil y poco resistente, sino que además se origina a costa de una consumición del refuerzo (10); 2) se hidrata y disuelve en ambientes acuosos, por lo que reduce la resistencia a la corrosión ambiental del AMC (11); 3) la reacción $<1>$ forma $\mathrm{Si}$, lo que fragiliza aún más el material compuesto resultante.

En el presente trabajo
TABLA I: CONDICIONES Y TIPO DE MATERIALES EMPLEADOS EN LOS ENSAYOS

\begin{tabular}{|c|c|c|c|c|}
\hline Aleación & $\begin{array}{c}\text { Contenido en } \mathrm{Si} \\
(\% \text { en peso) }\end{array}$ & $\begin{array}{l}\text { Partículas } \\
\text { SiC }\end{array}$ & $\begin{array}{c}\text { Temperatura } \\
\left({ }^{\circ} \mathrm{C}\right)\end{array}$ & $\begin{array}{c}\text { Tiempo } \\
\text { (min) }\end{array}$ \\
\hline AA1070 & - & recepción & 900 & 20 \\
\hline AA1070 & - & recepción & 900 & 60 \\
\hline A-319.0 & 6 & recepción & 900 & 20 \\
\hline A-319.0 & 6 & recepción & 900 & 60 \\
\hline A-332.0 & 11 & recepción & 900 & 20 \\
\hline A-332.0 & 11 & recepción & 900 & 20 \\
\hline AA1170 & - & $\begin{array}{c}\text { oxidadas } \\
\left(2 \mathrm{~h} \text { a } 1200^{\circ} \mathrm{C}\right)\end{array}$ & 900 & 20 \\
\hline AA1170 & - & $\begin{array}{c}\text { oxidadas } \\
\left(2 \mathrm{~h} \text { a } 1200^{\circ} \mathrm{C}\right)\end{array}$ & 900 & 60 \\
\hline AA1170 & - & $\begin{array}{c}\text { oxidadas } \\
\left(8 \mathrm{~h} \text { a } 1200^{\circ} \mathrm{C}\right)\end{array}$ & 900 & 20 \\
\hline AA1170 & - & $\begin{array}{c}\text { oxidadas } \\
\left(8 \mathrm{~h} \text { a } 1200^{\circ} \mathrm{C}\right)\end{array}$ & 900 & 60 \\
\hline
\end{tabular}
DE REACTIVIDAD ENTRE Al y PARTículas DE SIC. se estudia el desarrollo de un método simple para proteger a las partículas de SiC del ataque del aluminio fundido durante el procesado del material. Dicho método consiste en generar, sobre la superficie del refuerzo, una capa de $\mathrm{SiO}_{2}$ por oxidación. Para ello se analiza el comportamiento de partículas de $\mathrm{SiC}$ en estado de recepción y preoxidadas, frente a la acción de diferentes aleaciones de aluminio en estado fundido. Se realiza, asimismo, un estudio comparativo respecto al comportamiento de las condiciones de procesado de un material compuesto $\mathrm{Al} / \mathrm{SiC} p$ real.

\section{PROCEDIMIENTO EXPERIMENTAL}

\subsection{Materiales utilizados}

Los estudios realizados en el presente trabajo tuvieron como base la realización de ensayos de mojado entre partículas de $\mathrm{SiC}$ y diferentes aleaciones de aluminio. Para ello se emplearon tres tipos de aleaciones de aluminio: AA1170 (99,7\% Al), A-319.0 (Al-6Si-3Cu) y A-332.0 (Al-11Si-1,5Cu) que se mezclaron, una vez molidas y tamizadas, con una cantidad constante (30 \% vol.) de partículas de SiC. El SiC utilizado fue suministrado por Navarro S.A $(99,75 \%$ pureza) y poseía una granulometría F-360 (tamaños medios de $26,2 \mu \mathrm{m}$ ). Se prepararon pastillas compuestas de $\mathrm{Al} / \mathrm{SiC} p$ por prensado de los polvos, que posteriormente fueron sometidas a fusión en un horno de vacío.

Para la preparación de las pastillas prensadas se utilizaron tanto partículas en estado de recepción como otras sometidas a un proceso de oxidación controlada. El tratamiento de oxidación consistió en un calentamiento a $1200{ }^{\circ} \mathrm{C}$ en aire durante 2 y $8 \mathrm{~h}$. El espesor aproximado de la capa de óxido fue determinado a partir de la diferencia de pesada antes y después del tratamiento de oxidación.

\section{2.- Ensayos de fusión}

Con el fin de observar la reacción química interfacial a temperaturas superiores al punto de fusión de la matriz, se realizaron diferentes ensayos de fusión controlados sobre las muestras de material compuesto. Dichos ensayos simulaban los procesos de fabricación por colada típicos de AMCs. Por esta razón, las condiciones de temperatura $y$ tiempo de calentamiento seleccionadas se aproximaban a las empleadas en este tipo de procesos. Los ensayos de fusión se realizaron en un crisol de alúmina, produciendo la fusión y posterior solidificación en un horno de vacío $\left(\mathrm{Po}_{2}=2 \cdot 10^{-3} \mathrm{~Pa}\right)$. El ciclo de 
fusión constaba de una etapa de calentamiento lento hasta la temperatura de fusión en la que era mantenido un tiempo variable. En la tabla I se recogen las condiciones de dichos ensayos.

Para verificar la similitud de los fenómenos interfaciales analizados en los materiales preparados por el procedimiento anteriormente expuesto y los ocurridos en un material compuesto real sometido a las mismas condiciones de fusión, se empleó una aleación de aluminio AA2014 (Al-4,3Cu-0,12Mg) reforzada con un $13 \%$ de partículas de $\mathrm{SiC}$. Este material, recibido en forma de chapón extruido, de $20 \mathrm{~mm}$ de espesor, fue sometido también a los ensayos de fusión aplicados sobre las mezclas de partículas de $\mathrm{SiC}$ y polvos de aluminio.

\subsection{Preparación de muestras y técnicas experimentales}

Las partículas de $\mathrm{SiC}$ (oxidadas y no) y los polvos metálicos fueron caracterizados utilizando difracción de rayos $\mathrm{X}(\mathrm{DRX})$, microscopía electrónica de barrido (MEB) y de transmisión (MET), junto con difracción de electrones (DE). También los materiales compuestos preparados se estudiaron microestructuralmente mediante MEB y MET. En este caso, las muestras se prepararon siguiendo un procedimiento convencional de desbaste y pulido, pero evitando el contacto con agua, para preservar al máximo el $\mathrm{Al}_{4} \mathrm{C}_{3}$ formado. Se empleó de nuevo la técnica de DRX, con objeto de determinar las fases formadas durante a los ensayos de fusión y su evolución en función de las condiciones de ensayo.

\section{RESULTADOS Y DISCUSIÓN}

\subsection{Influencia del tratamiento de fusión sobre el material compuesto AA2014/SiC/13p}

La figura 1 muestra la microestructura típica que posee un material compuesto de matriz de aluminio obtenido por vía pulvimetalúrgica seguida de extrusión. En este caso, se trata de la aleación AA2014, reforzado con un $13 \%$ de partículas de SiC. El proceso de fabricación seguido origina una distribución en bandas del refuerzo y minimiza la reacción interfacial entre la matriz y las partículas. Esto queda de relieve cuando el material se estudia con técnicas de alto poder de resolución (MET), apreciándose como la interfase matriz/partícula se caracteriza por encontrarse libre de productos de reacción, no habiéndose observado, en ningún caso, la presencia de carburo de aluminio (fig. 2).

En este tipo de materiales, es frecuente encontrar diferentes tipos de $\mathrm{SiC}(\alpha-\operatorname{SiC} 33 \mathrm{R}, \alpha-\mathrm{SiC} 6 \mathrm{H})$, que presentarían a priori un comportamiento químico también diferente. La figura 2 muestra un fragmento de una partícula de $\mathrm{SiC}$, cuya microdifracción de electrones demostró que se trataba de $\alpha-S i C$ 33R hexagonal $\left(a_{0}=\right.$ 3,073 А y $\mathrm{c}_{\mathrm{o}}=82,94 \AA$.).

Cuando un material de esta naturaleza sufre un proceso de fusión de su matriz experimenta una serie de reacciones interfaciales que originan la formación de productos que degradan al material y que dependen de las condiciones de temperatura y tiempo en las que se produce la fusión. Los estudios de difracción de rayos-

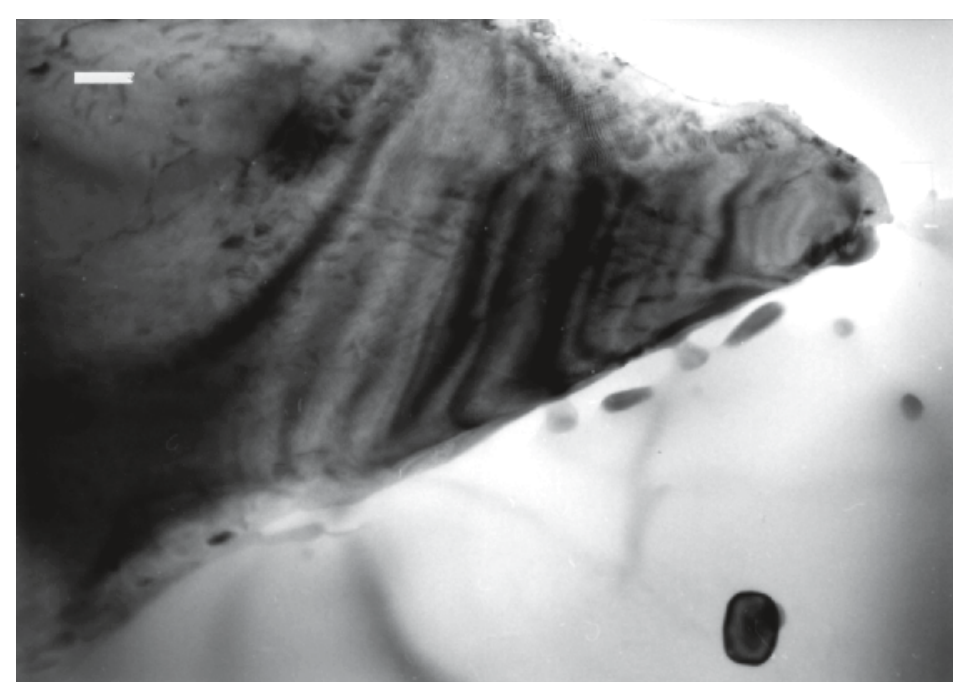

Fig. 2: Intercara AA 2014/SiC/13p en estado de recepción. (barra: 100 nm).
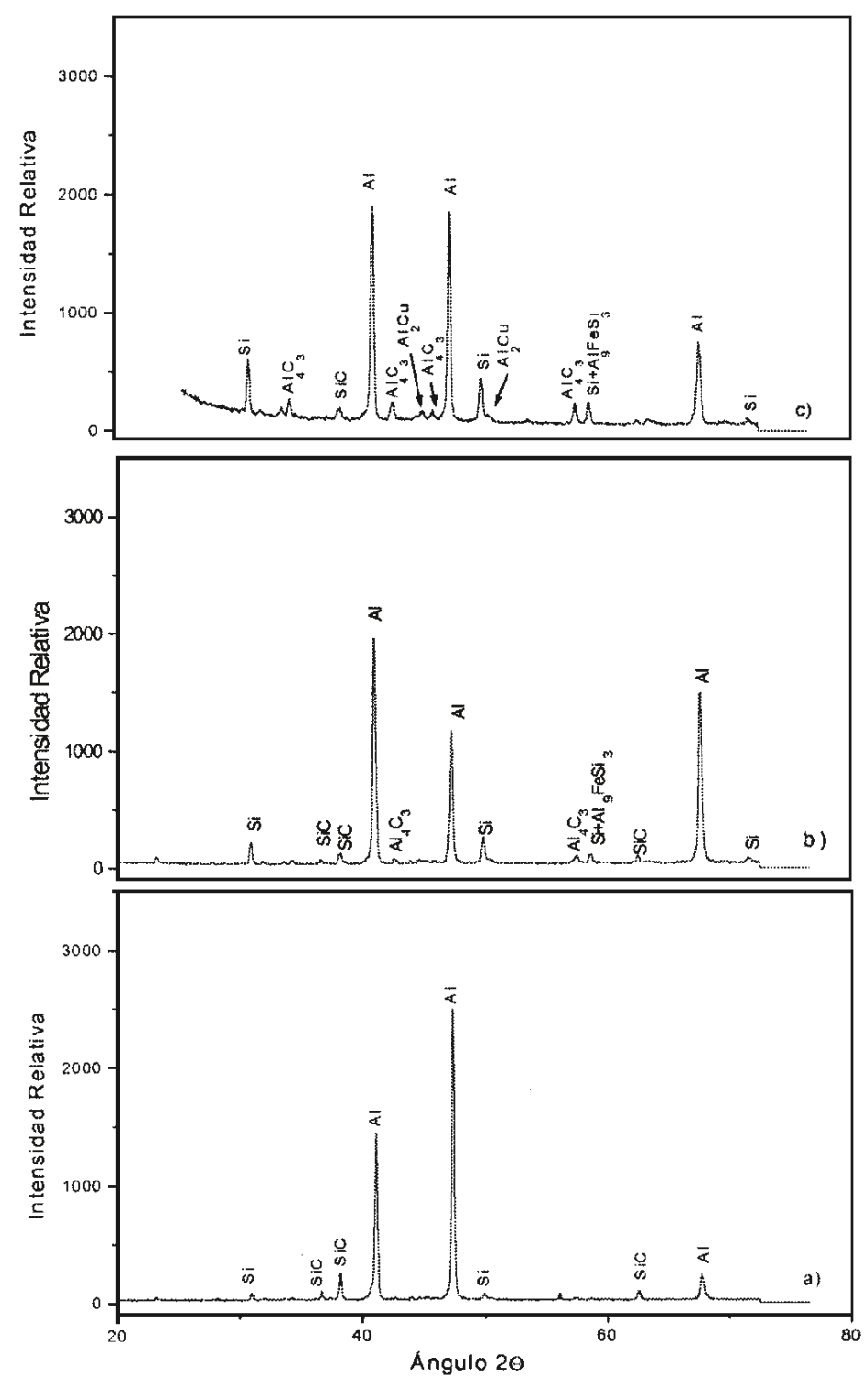

Fig. 3: Difracción de rayos- $X$ : a) material base, b) material ensayado a $750^{\circ} \mathrm{C}$ y c) material ensayado a $850^{\circ} \mathrm{C}$. 

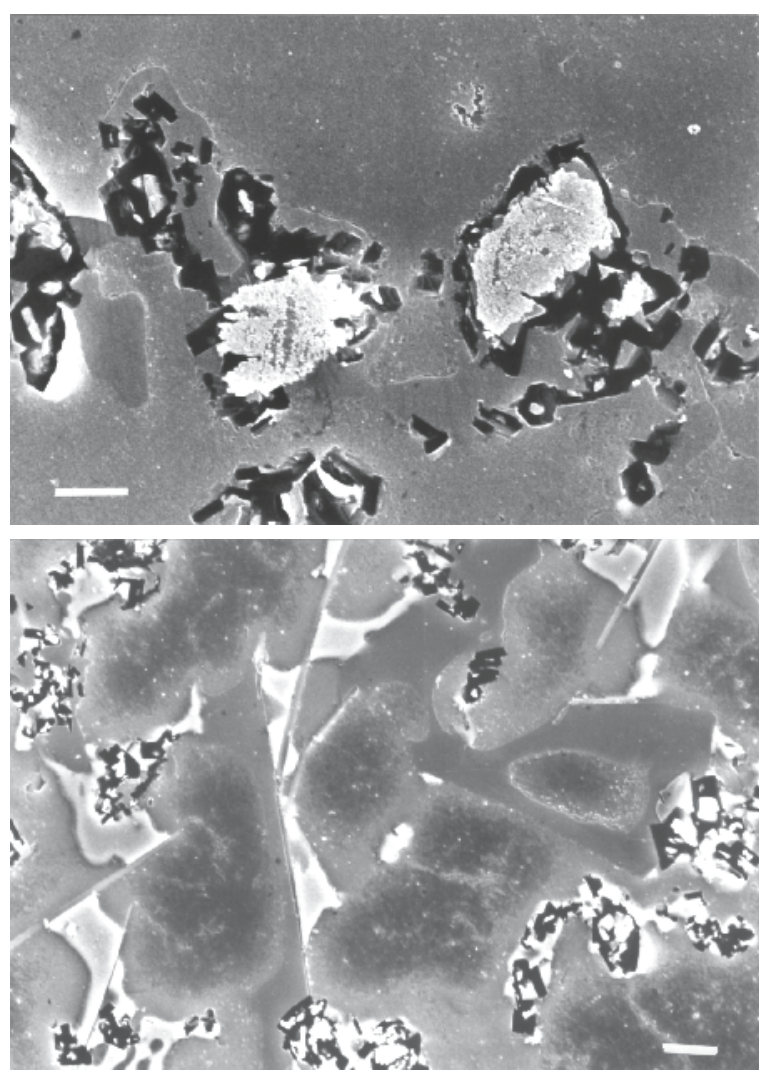

Fig.4: a) Reacción alrededor de las partículas. (barra: $5 \mathrm{~mm}$ ) b) Microestructura del material fundido a $850^{\circ} \mathrm{C}, 30$ minutos. (barra: $10 \mu \mathrm{m})$.

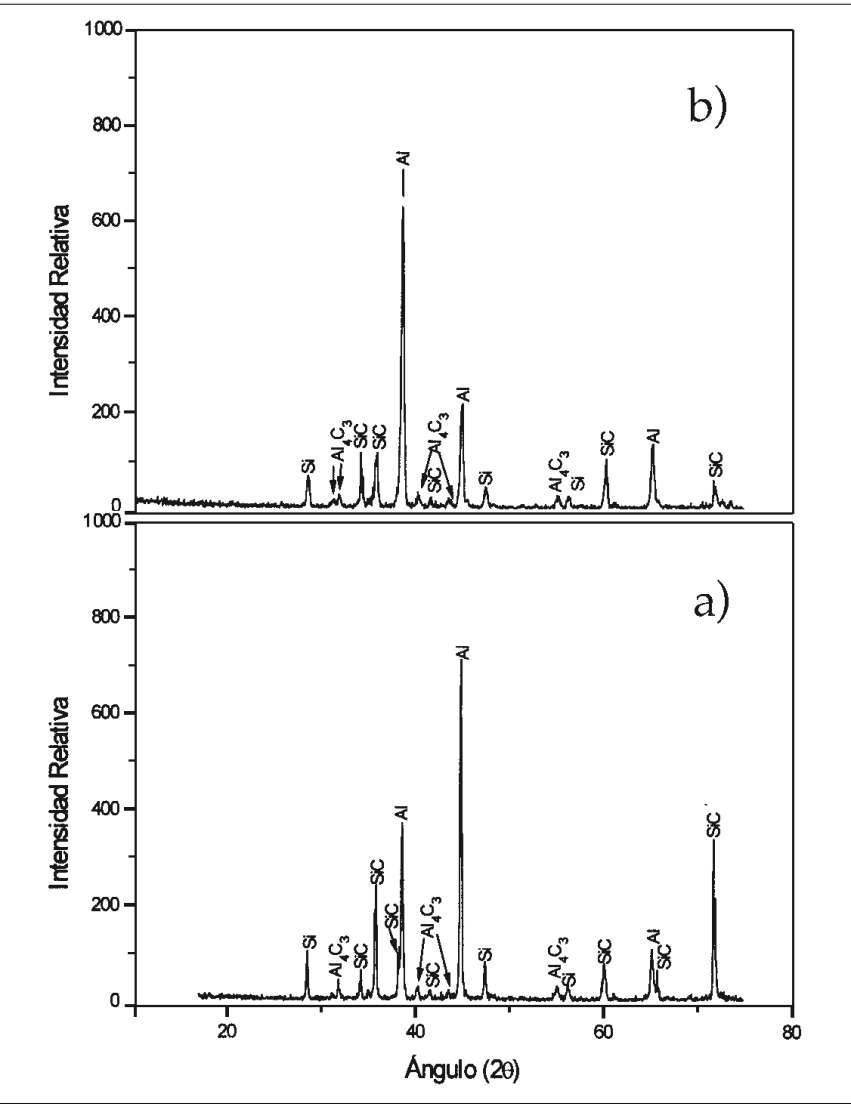

Fig. 6: Difración de rayos- $\mathrm{X}$ de los materiales $\mathrm{Al} / \mathrm{SiCp}$ ensayados, a) $900^{\circ} \mathrm{C}, 20$ minutos y b) $900^{\circ} \mathrm{C}, 60$ minutos.

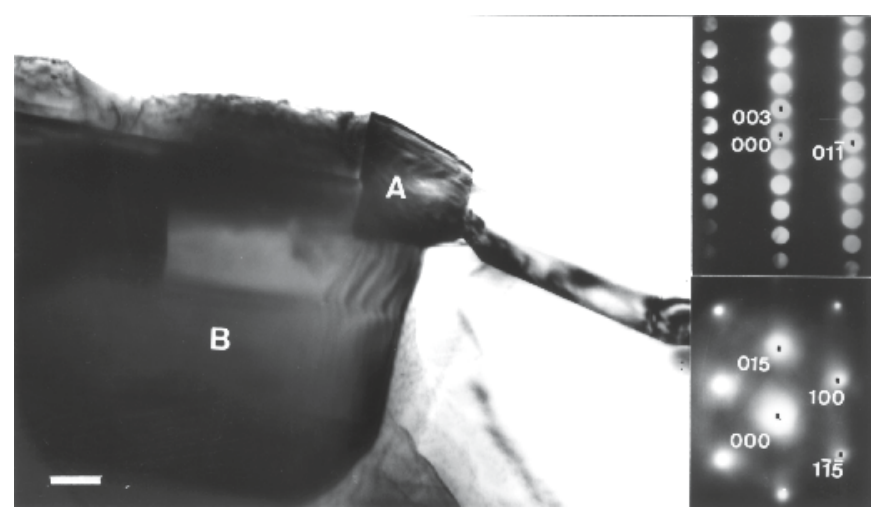

Fig. 5: Formación de $\mathrm{Al}_{4} \mathrm{C}_{3}$ (A) sobre SiCp (B), (barra: $200 \mathrm{~nm}$ ) b) difracción de electrones sobre $\mathrm{Al}_{4} \mathrm{C}_{3}$, c) difracción de electrones sobre $\mathrm{SiCp}$.

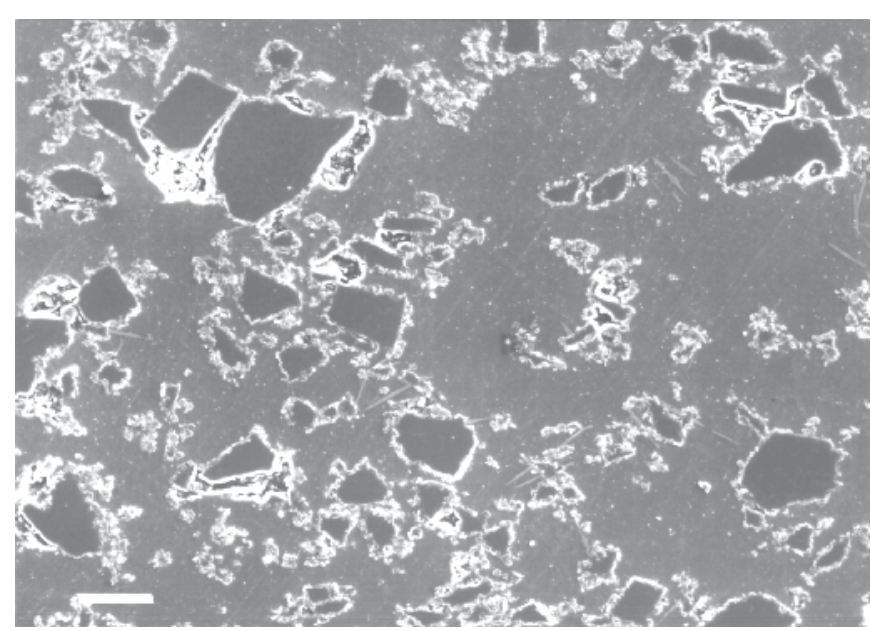

Fig. 7: Microestructura del material $\mathrm{Al} / \mathrm{SiCp}$ ensayado a $900^{\circ} \mathrm{C}$ durante 20 minutos. (barra: $50 \mu \mathrm{m}$ ).

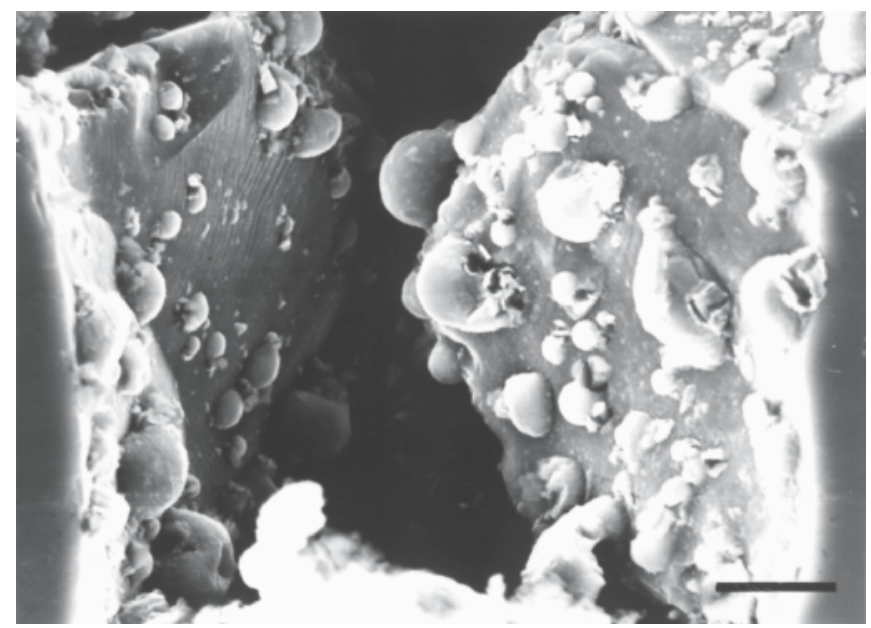

Fig. 8: Zona de porosidad entre partículas de SiC. (barra: $10 \mu \mathrm{m}$ ). 


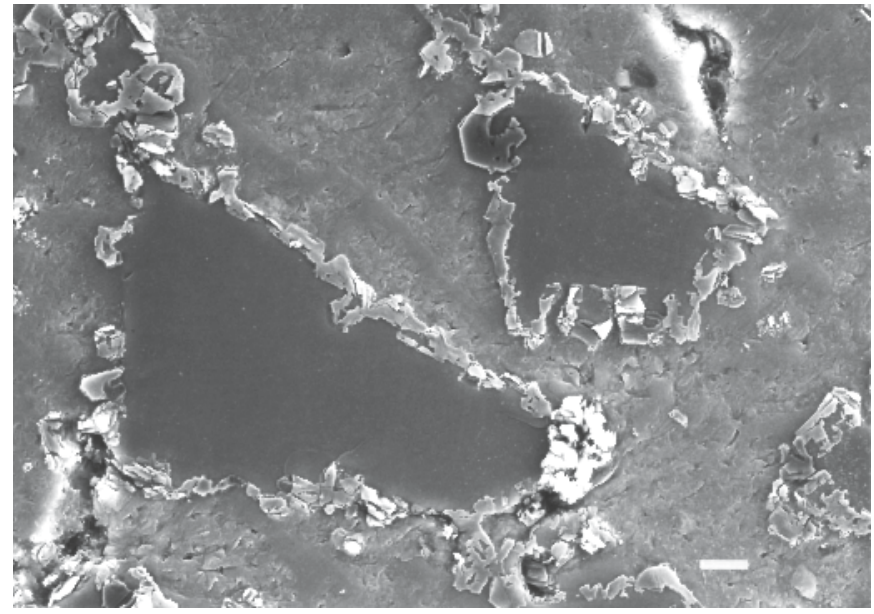

Fig. 9: Reacción interfacial en $\mathrm{Al} / \mathrm{SiCp}$ ensayado a fusión a $900^{\circ} \mathrm{C}, 60$ minutos. (barra: $5 \mu \mathrm{m}$ ).

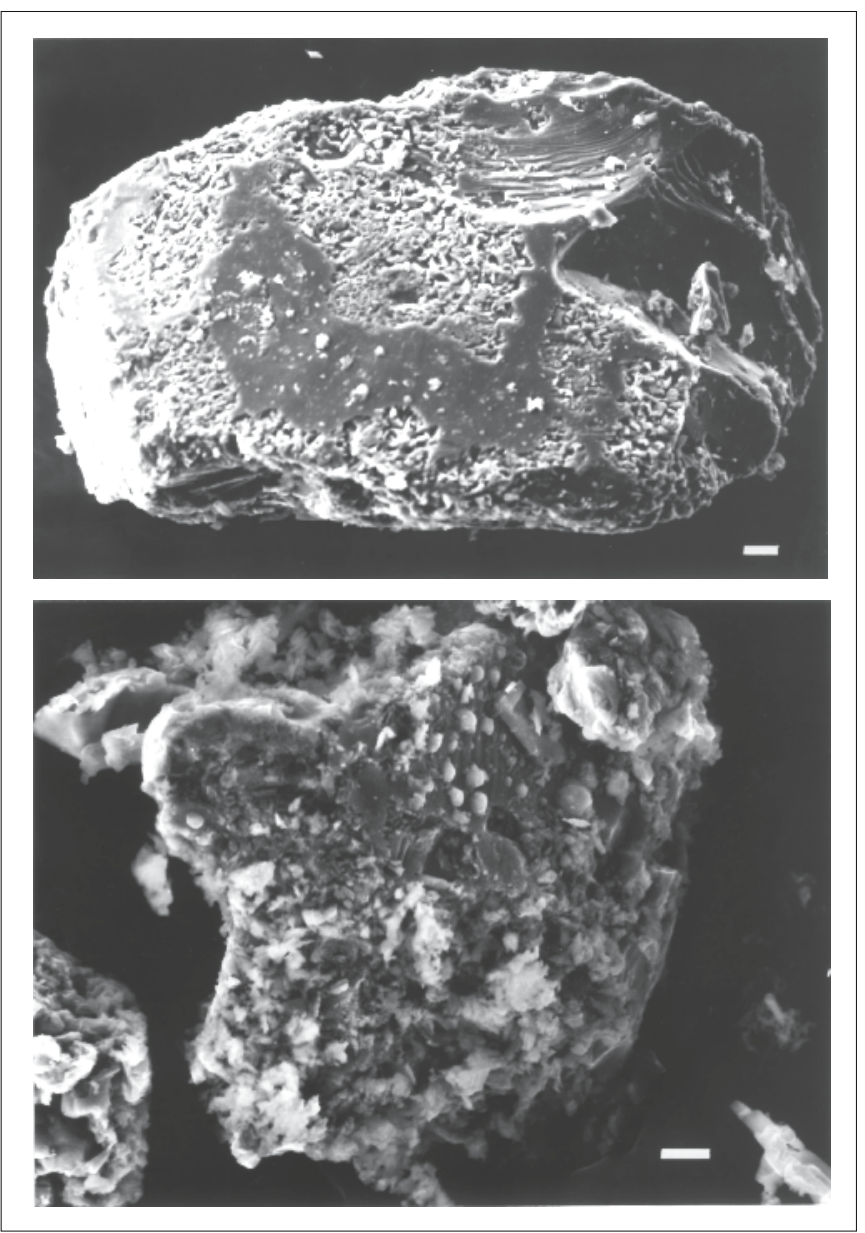

Fig. 10: Ataque sobre las partículas de SiC después de ensayadas a $900^{\circ} \mathrm{C}$. a) 20 minutos (Barra: $10 \mu \mathrm{m}$ ); b) 60 minutos (barra: $5 \mu \mathrm{m}$ ).

X (fig. 3), realizados en muestras del material AA2014/ $\mathrm{SiC} / 13 p$ fundidas y posteriormente solidificadas en diferentes condiciones, muestran que el incremento de temperatura y tiempo de fusión producen la formación gradual de $\mathrm{Al}_{4} \mathrm{C}_{3}$ y Si (este elemento se combina inicialmente con las impurezas de Fe presentes en la matriz, para formar la fase ternaria $\mathrm{Al}_{9} \mathrm{FeSi}_{3}$ ), a costa del consumo de las partículas de refuerzo.

El estudio microestructural de los materiales así tratados pone de relieve que, aunque a temperaturas de $750^{\circ} \mathrm{C}$, la for- mación de $\mathrm{Al}_{4} \mathrm{C}_{3}$ se produce en ciertas zonas preferentes de la partícula como muestra figura 4.a (fase oscura); el aumento del tiempo de permanencia en estado fundido ( $>20 \mathrm{~min}$ ) o de la temperatura produce el consumo casi total de las partículas de refuerzo (fig. 4.b) unido a la formación de una elevada proporción de fases intermetálicas ricas en silicio.

La observación por MET de las intercaras SiC/matriz después de este tipo de tratamiento ha permitido comprobar que, ya a $750^{\circ} \mathrm{C}$ y 20 minutos de fusión, se forman agregados cristalinos de $\mathrm{Al}_{4} \mathrm{C}_{3}$ por la reacción $<1>$, en ciertos lugares preferentes de las partículas de SiC (fig. 5). En este caso, los carburos de aluminio forman cristales discretos, que se han identificado por difracción de electrones, creciendo no sólo en la matriz de aluminio sino también hacia el interior de la partícula de $\mathrm{SiC}$.

\subsection{Ensayos de fusión sobre pastillas compactadas de Al/ $\operatorname{SiCp}$}

\subsubsection{PARTÍCULAS NO OXIDADAS}

Las figuras 6a y b comparan dos difractogramas de rayos- $\mathrm{X}$ obtenidos sobre dos pastillas de la aleación AA1170 con un $30 \%$ en volumen de partículas de $\mathrm{SiC}$, después de ser sometidas a $900{ }^{\circ} \mathrm{C}$ durante 20 y $60 \mathrm{~min}$. Como hecho más significativo, se aprecia la aparición de los picos correspondientes a $\mathrm{Al}_{4} \mathrm{C}_{3}$, cuya intensidad se incrementa con el tiempo de fusión. También se detecta un importante aumento de la intensidad del pico del Si, elemento que no está inicialmente presente en este tipo de aleación y que procede de la reacción interfacial ya comentada. En cambio, la intensidad relativa entre los picos de los dos elementos mayoritarios ( $\mathrm{Al}$ y $\mathrm{SiC}$ ) está condicionada por la distribución de ambos en la superficie difractada. Las condiciones de mezcla empleadas y la posterior redistribución del refuerzo durante la fusión de la probeta solían originar distribuciones heterogéneas entre ambos constituyentes.

El estudio microestructural de estos materiales demuestra como ya a $900^{\circ} \mathrm{C}$, durante 20 minutos, se produce mojado y la reacción química en las intercaras matriz/partícula está bastante extendida (fig. 7). Se pueden observar también algunas zonas de porosidad favorecidas por la acumulación local de partículas (fig. 8), en las que se distinguen gotas de aluminio solidificadas sobre las superficies de las partículas de $\mathrm{SiC}$. Se aprecia cómo, en las zonas de contacto $\mathrm{Al} / \mathrm{SiC}$, se han formado pequeños cristales de productos de reacción $\left(\mathrm{Al}_{4} \mathrm{C}_{3}\right)$.

Aumentando el tiempo de fusión a $60 \mathrm{~min}$, el consumo del refuerzo cerámico está más favorecido (fig. 9), y las partículas se encuentran completamente rodeadas de productos de reacción, de forma similar a lo que ocurría en el material compuesto sometido al proceso de fusión a vacío.

Con el fin de observar cómo se producía el ataque del aluminio fundido sobre las partículas de $\mathrm{SiC}$, sobre estas mismas muestras se realizó una extracción de la fase cerámica. Para ello se procedió a la disolución electrolítica de la matriz con $\mathrm{HNO}_{3}$ ( $30 \% \mathrm{vol})$, y posterior filtrado del residuo sólido. En la figura 10a y b, se muestran dos de las partículas de SiC extraídas a partir de muestras calentadas a $900{ }^{\circ} \mathrm{C}$ durante 20 y $60 \mathrm{~min}$, respectivamente. En ambos casos, se observan las zonas de ataque sobre el $\mathrm{SiC}$ y cómo, en el segundo caso, el ataque está más generalizado sobre la toda la superficie de la partícula y se observa más proporción de cristales de $\mathrm{Al}_{4} \mathrm{C}_{3}$ sobre ella.

La reacción interfacial observada puede ser controlada, según la ecuación $<2>$, aumentando la actividad de silicio libre en la matriz. Por este motivo, se realizaron ensayos de 

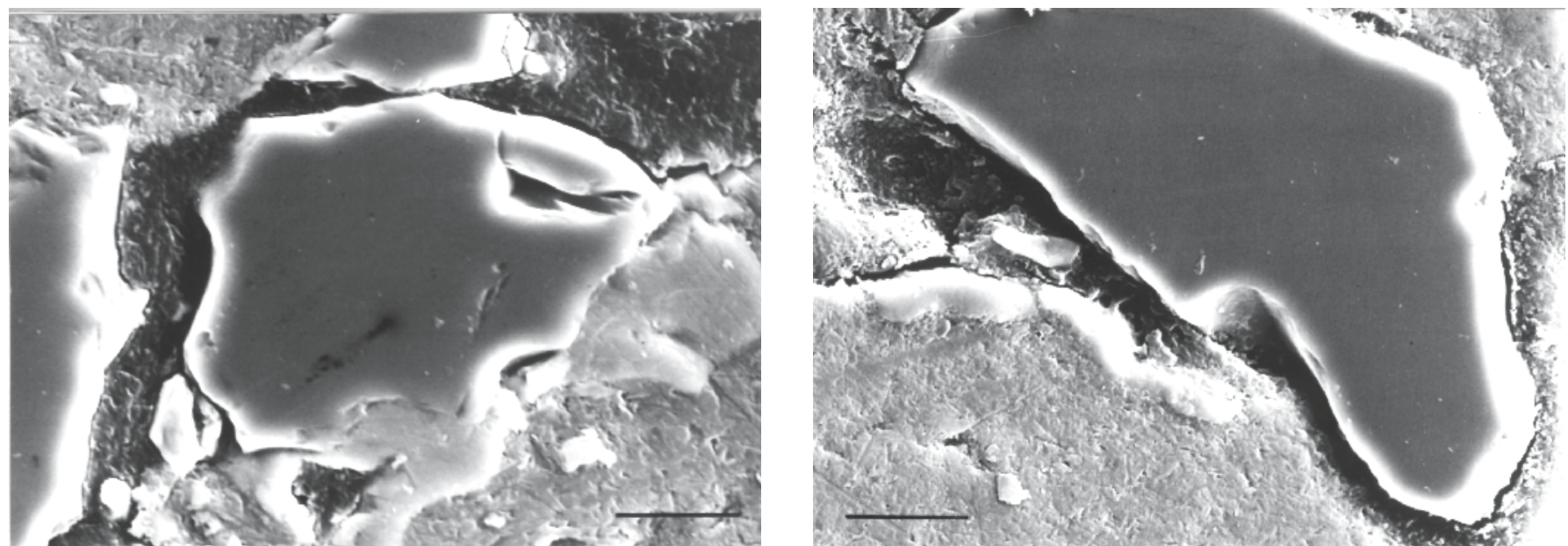

Fig. 11: Microestructura de los materiales compuestos fabricados con polvos de aleaciones de Al-Si: a) Al-6Si-3Cu (barra: $10 \mu \mathrm{m})$; b) Al-11Si-1,5Cu (barra: $10 \mu \mathrm{m}$ ).

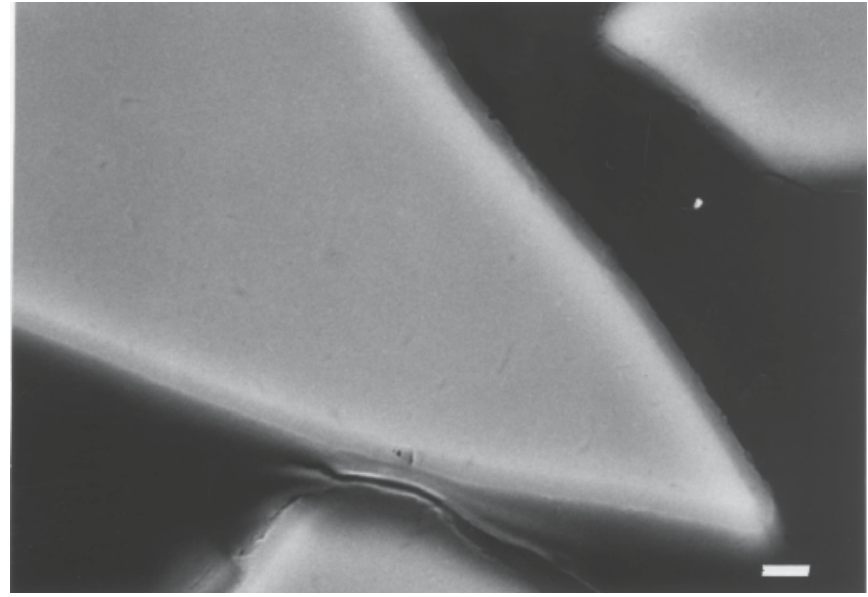

Fig. 12: Imagen de electrones retrodifundidos de partículas de $\mathrm{SiC}$ oxidadas a $1200{ }^{\circ} \mathrm{C}(2 \mathrm{~h})$ con formación de una capa continua de óxido (barra: $1 \mu \mathrm{m}$ ).
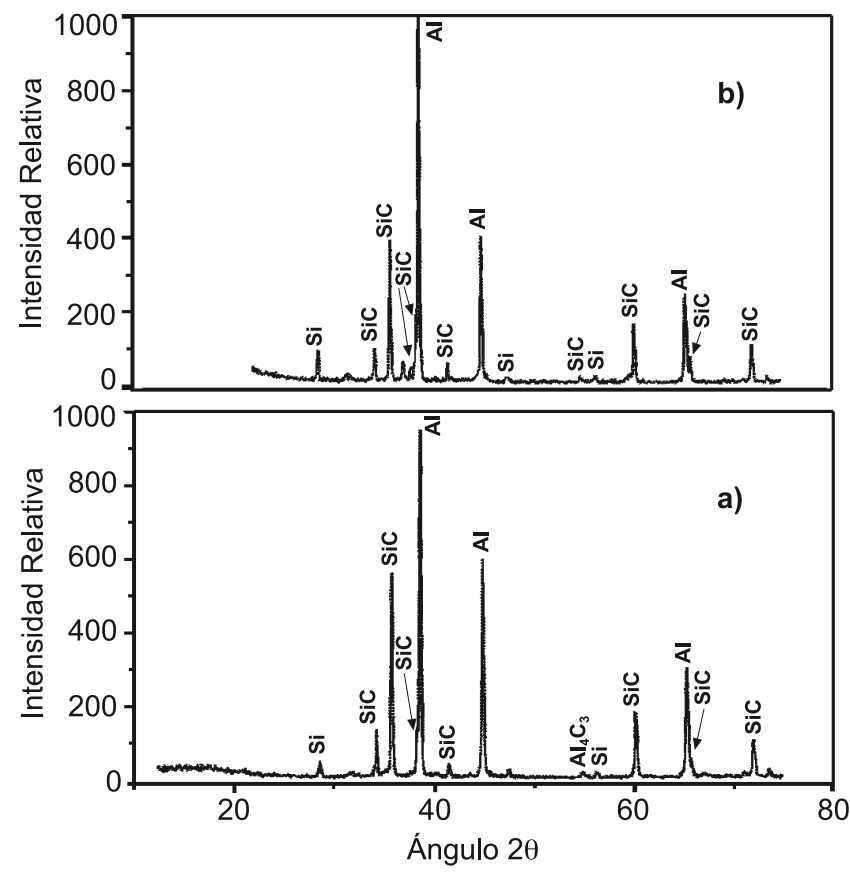

Fig. 13: Difracción de rayos- $\mathrm{X}$ en materiales $\mathrm{Al} / \mathrm{SiC}$ oxidado después de ensayos a fusión a $900{ }^{\circ} \mathrm{C}$, a) $20 \mathrm{~min}$. b) $60 \mathrm{~min}$. mojado-reactividad con aleaciones de aluminio de altos contenidos en $\mathrm{Si}$ (6\% y $13 \%$ ). El resultado, en ambos casos fue que, si bien no se detecta la formación de $\mathrm{Al}_{4} \mathrm{C}_{3}$ en las intercaras $\mathrm{SiC} / \mathrm{Al}$ se ha reducido de forma notable la mojabilidad entre ambos constituyentes, tal y como se aprecia en las microestructuras recogidas en las figuras 11a y b. Este resultado demuestra que existe una correlación aparente entre la mojabilidad y la reactividad y que, aunque la reacción de formación de $\mathrm{Al}_{4} \mathrm{C}_{3}$ es altamente perjudicial para el material compuesto resultante, la existencia de una reacción en la intercara metal/cerámico reduce su energía interfacial, aumentando la mojabilidad.

\subsubsection{PARTÍCULAS OXIDADAS}

El tratamiento de oxidación aplicado sobre las partículas de $\mathrm{SiC}$ genera una capa de $\mathrm{SiO}_{2}$ que está constituida fundamentalmente por cristobalita como se demostró mediante DRX. Los cálculos realizados a partir de la ganancia de peso sufrida por las partículas durante el proceso de oxidación, considerando que se formaban capas de homogéneas de $\mathrm{SiO}_{2}$, permitieron estimar que los espesores teóricos obtenidos eran aproximadamente 100 y $136 \mathrm{~nm}$, para las dos condiciones de oxidación empleadas. La figura 12 muestra un detalle en superficie pulida (MEB-electrones retrodifundidos) de las partículas oxidadas antes de su incorporación a la aleación de aluminio (AA1170), donde se comprueba la continuidad de la capa de óxido formada.

Los ensayos de reactividad realizados con este tipo de partículas, en las mismas condiciones que las aplicadas a las partículas no oxidadas, muestran una importante reducción del nivel de reacción interfacial. Las difracciones de rayos $X$ (fig. 13) realizadas en estos materiales muestran que, aunque existe formación de $\mathrm{Al}_{4} \mathrm{C}_{3}$ y $\mathrm{Si}$, se encuentra mucho más limitada que en partículas no protegidas. En la figura 14, se muestra un detalle de la reducida reactividad que existe entre las partículas y la matriz de aluminio, a pesar de que la intercara entre ambas se formó a $900{ }^{\circ} \mathrm{C}$ durante $20 \mathrm{~min}$.

Otro de los hechos observados a partir de los ensayos con partículas preoxidadas es que, a pesar de inhibir la reacción interfacial de formación de $\mathrm{Al}_{4} \mathrm{C}_{3}$, no se aprecian problemas de mojabilidad como los detectados cuando se empleaban aleaciones de aluminio ricas en $\mathrm{Si}$, para desplazar la reacción $<1>$ 


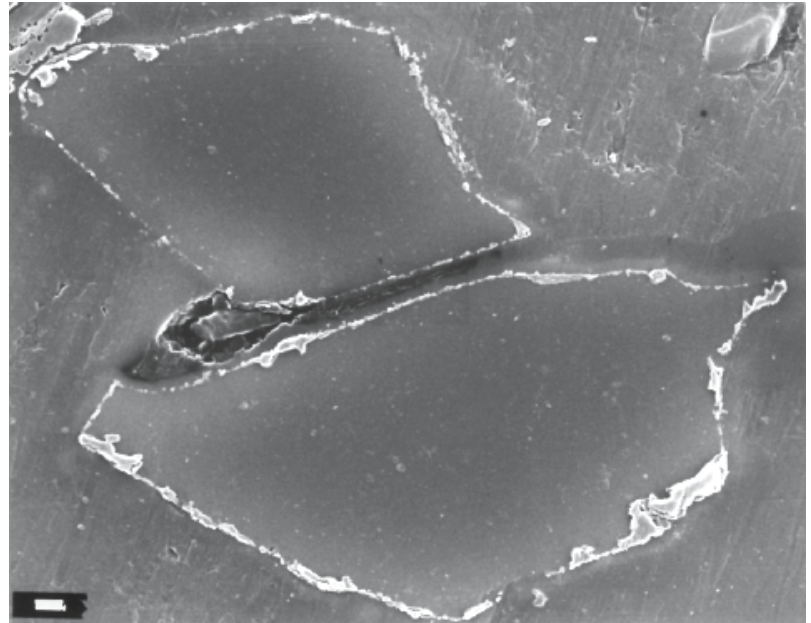

Fig. 14: Imagen MEB de la interacción interfacial entre $\mathrm{Al}$ fundido y partículas de $\mathrm{SiC}$ oxidadas $\left(1200{ }^{\circ} \mathrm{C}, 2 \mathrm{~h}\right)$ a $900{ }^{\circ} \mathrm{C}$ durante $20 \mathrm{~min}$. (barra: $1 \mu \mathrm{m}$ ).

hacia la derecha. Este comportamiento puede deberse a que la capa de $\mathrm{SiO}_{2}$ que protege a la partícula de $\mathrm{SiC}$ no es completamente inerte, sino que reacciona con el aluminio fundido, entre 700 y $900{ }^{\circ} \mathrm{C}$ formando $\mathrm{Al}_{2} \mathrm{O}_{3}$, según la reacción [12]:

$$
4 \mathrm{Al}+3 \mathrm{SiO}_{2} \rightarrow 2 \mathrm{Al}_{2} \mathrm{O}_{3}+3 \mathrm{Si}
$$

El estudio con MET de este tipo de intercaras (fig. 15) ha demostrado la formación de $\delta$-alúmina mediante la reacción $<3>$, junto con otras capas intermedias de Al-Si-O amorfo y mullita microcristalina, lo que apunta a la participación de otras reacciones interfaciales como son:

$$
\begin{array}{cc}
\mathrm{SiO}_{2}+12 / 13 \mathrm{Al} \rightarrow 2 / 13\left(3 \mathrm{Al}_{2} \mathrm{O}_{3} \cdot 2 \mathrm{SiO}_{2}\right)+9 / 13 \mathrm{Si} & <4> \\
x \mathrm{Al}+y \mathrm{SiO}_{2} \rightarrow \mathrm{Al}_{x} \mathrm{Si}_{y} \mathrm{O}_{z} & <5>
\end{array}
$$

La formación de $\mathrm{Al}_{2} \mathrm{O}_{3}$ en la intercara $\mathrm{Al} / \mathrm{SiC}$ no originaría los problemas mecánicos y de degradación química asociados a la formación de $\mathrm{Al}_{4} \mathrm{C}_{3}$. No obstante, y en las condiciones de reactividad ensayadas $\left(900{ }^{\circ} \mathrm{C}, 20-60 \mathrm{~min}\right.$ ) la formación de una capa protectora de $\mathrm{SiO}_{2}$, con espesores medios comprendidos entre 100 y $136 \mathrm{~nm}$ no evita totalmente la reacción entre el aluminio fundido y la partícula de $\mathrm{SiC}$, por lo que se deduce que dicha barrera se consume totalmente para los tiempos de colada ensayados. La ausencia de picos de $\mathrm{SiO}_{2}$ en las difracciones de rayos $\mathrm{X}$ adquiridas en ensayos de reactividad con partículas preoxidadas, demuestran este hecho.

\section{CONCLUSIONES.}

Los procesos convencionales de fabricación de aleaciones de aluminio por técnicas de colada no son aplicables cuando estas aleaciones están reforzadas con partículas de $\mathrm{SiC}$ por la formación de $\mathrm{Al}_{4} \mathrm{C}_{3}$ como consecuencia de la reacción interfacial entre el $\mathrm{SiC}$ y el aluminio fundido.

Aunque la reacción de formación de $\mathrm{Al}_{4} \mathrm{C}_{3}$ puede inhibirse mediante el empleo de aleaciones de aluminio ricas en silicio (A-319.0 y A-332.0), la ausencia de una reacción interfacial reduce la mojabilidad del aluminio fundido sobre el $\mathrm{SiC}$, lo que originaría problemas de fabricación por técnicas de mol-

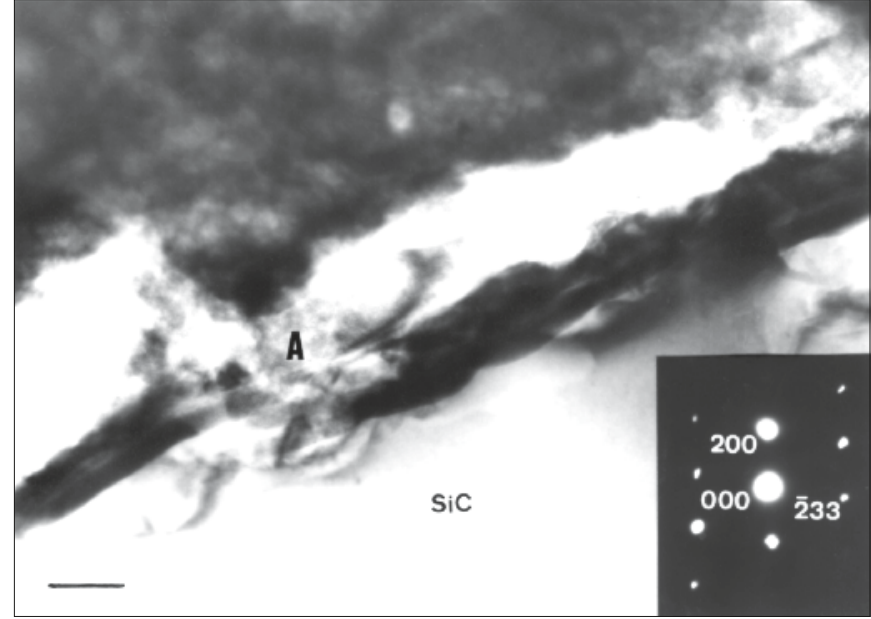

Fig. 15: Imagen MET de la formación de alúmina (DE, eje de zona [0 1 i 1 $]$ ) en la intercara entre $\mathrm{SiC}$ oxidado $\left(1200{ }^{\circ} \mathrm{C}, 8 \mathrm{~h}\right)$ y Al fundido (900 ${ }^{\circ} \mathrm{C}, 1 \mathrm{~h}$ ). (barra: $100 \mathrm{~nm}$ )

deo.

La introducción de una capa de óxido $\left(\mathrm{SiO}_{2}\right)$ sobre la superficie de las partículas $\mathrm{SiC}$ por oxidación directa, parece jugar un papel importante en la prevención del ataque del SiC por el aluminio líquido, sin que se observe reducción en la mojabilidad del $\mathrm{SiC}$, como ocurre cuando se emplean aleaciones de aluminio ricas en $\mathrm{Si}$.

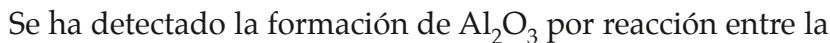
capa de $\mathrm{SiO}_{2}$ formada sobre las partículas de $\mathrm{SiC}$ y el aluminio formado, junto con otros compuestos intermedios Al-Si-O (aluminosilicatos amorfos, mullita).

En las condiciones de mojado empleadas (900 ${ }^{\circ} \mathrm{C}$, 20-60 min) y para recubrimientos continuos de $\mathrm{SiO}_{2}$ con espesores medios de 100 a $136 \mathrm{~nm}$, no se llega a inhibir completamente la formación de $\mathrm{Al}_{4} \mathrm{C}_{3}$, al consumirse completamente la barrera de protección por reacción con el aluminio fundido.

\section{BIBLIOGRAFIA}

1. D.J. Lloyd. "Particle reinforced aluminium and magnesium matrix composites". Int. Mat. Rev. 39 (1) 1-22 (1994).

2. S.J. Harris. "Developments in particulate and short fibre composites". Agard Lectures Series No. 174 (New Light Alloys). AGARD-NATO. 4-1/4-21 (1990).

3. P.G. Partridge, C.M. Ward-Close. "Processing of advanced continuous fibre composites: Current practice and potential developments". Int. Mat. Rev. 38 (1) 1-24 (1993).

4. W.M. Zhong, G. L'Espérance, M. Suéry. Interfacial reactions in Al-Mg (5083)/ SiCp composites during fabrication and remelting. Met. \& Mat. Trans. A. 26A (10)2637-2649 (1995).

5. T.W. Clyne "Metallic composite materials" en "Physical Metallurgy" $4^{\text {th }}$ Edition (Ed. R.W. Cahn \& P. Haasen). Elsevier Science BV. Cap. 30, 25682622 (1996).

6. R.R. Kieschke, C.M. Warwick, T.W. Clyne. "Sputter deposited barrier coatings on SiC monofilaments for use in reactive metallic matrices- Part I. Optimization of barrier structure". Acta Met. et Mat. 39, 427-436 (1991).

7. T.W. Clyne, a.J. Phillipps. "Interfacial control and macroscopic failure in long-fibre-reinforced and laminated inorganic composites", Comp. Sci. \& Tech. 51 271-282 (1994).

8. V. Laurent, D. Chatain, N. Eustathopolous. “Wettabity of SiC by aluminium and AlSi alloys". J. Mater. Sci. 22, 244-250 (1987).

9. A.S. Isaikin, V.M. Chubarov, B.F. Tretilov, V.A. Silaer, Y.A. Goselor. Mat. Sci. Heat. Treat. (Russia) 22 (11-22). 815-817 (1980). 
10. D.S. Shin, J.C. Lee, E.P Yooh, H.I. Lee. "Effect of the processing methods on the formation of $\mathrm{Al}_{4} \mathrm{C}_{3}$ in $\mathrm{SiCp} / 2024 \mathrm{Al}$ composites". Mat. Res. Bull. 32 (9) 1155-1163 (1997).

11. J.K. Park, J.P. Lucas. “Moisture effect on SiCp/6061/Al MMC: dissolution of interfacial $\mathrm{Al}_{4} \mathrm{C}_{3}$. Script. Mat. 37 (4) 511-516 (1997)

12. J. Narciso, A. Alonso, C. García-Cordobilla, A. Paimes, E. Louis. “Reactivity ans wettability of thermally oxidized and unoxidized $\mathrm{SiC}$ particulates with aluminium, and its effects on composite fabrication" en "MetalMatrix Composites, ICCM9" Vol. I Ed. A. Miravete. Woodhead Publ. Ltd. Zaragoza 779-786 (1993).

\section{AGRADECIMIENTOS:}

Los autores del presente trabajo desean agradecer a la CICYT (Proyecto MAT97/0719) el apoyo económico brindado para su posible realización y a Navarro S.A. por la cesión de las partículas de SiC empleadas en el presente estudio.

Recibido: 21-10-98

Aceptado: 7-5-99 